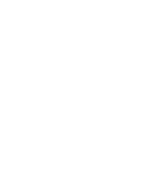
ANNUAL Further
REVEWS

Click here for quick links to Annual Reviews content online, including:

- Other articles in this volume

- Top cited articles

- Top downloaded articles

- Our comprehensive search

\section{Optimal Vortex Formation as a Unifying Principle in Biological Propulsion}

\section{John O. Dabiri}

Graduate Aeronautical Laboratories and Bioengineering, California Institute of Technology, Pasadena, California 91125; email: jodabiri@caltech.edu

\section{Key Words}

First published online as a Review in Advance on June 3, 2008

The Annual Review of Fluid Mechanics is online at fluid.annualreviews.org

This article's doi:

10.1146/annurev.fluid.010908.165232

Copyright (C) 2009 by Annual Reviews. All rights reserved

$0066-4189 / 09 / 0115-0017 \$ 20.00$ hydrodynamics, aerodynamics, cardiovascular flows, swimming, flying

\section{Abstract}

I review the concept of optimal vortex formation and examine its relevance to propulsion in biological and bio-inspired systems, ranging from the human heart to underwater vehicles. By using examples from the existing literature and new analyses, I show that optimal vortex formation can potentially serve as a unifying principle to understand the diversity of solutions used to achieve propulsion in nature. Additionally, optimal vortex formation can provide a framework in which to design engineered propulsions systems that are constrained by pressures unrelated to biology. Finally, I analyze the relationship between optimal vortex formation and previously observed constraints on Strouhal frequency during animal locomotion in air and water. It is proposed that the Strouhal frequency constraint is but one consequence of the process of optimal vortex formation and that others remain to be discovered. 
Vortex formation time: the dimensionless timescale during which vortex formation occurs

\section{INTRODUCTION}

With the exception of swimming microorganisms, locomotion in water and air occurs in the Reynolds number regime $R e>10^{2}$ (based on characteristic appendage length and velocity), in which the formation and shedding of coherent vortices are predominant. Similarly, many internal flows in biological systems are characterized by flow separation and subsequent vortex shedding. Blood flow in the human heart is a common example. Despite the broad range of biological systems exhibiting vortex formation and shedding, physical constraints on the kinematics of vortex lines (i.e., Helmholtz's laws) are sufficiently severe that only a few vortex motifs are found in flows containing free vorticity. Closed loops of vorticity_vortex rings—and derivatives thereof are a consistent feature of biological propulsion; indeed, vortex rings may be the only consistent feature. Given this observation, I ask whether the kinematic and dynamic properties of vortex rings may provide a unifying perspective from which to examine the diversity of solutions used in nature to achieve effective transport and transfer mass, momentum, and energy in fluids.

This discussion is motivated in part by recent efforts by engineers and biologists to synthesize strategies for optimal propulsion (whose definition is made precise below) from the wealth of observations of animals now available. Notwithstanding, I do not take a position that anticipates intrinsic fluid dynamic optimality in biological propulsion systems. The evolution of biological propulsion occurs in a landscape constrained by factors partly or wholly unrelated to propulsion per se (e.g., phylogeny, physiology, reproduction, metabolism, and communication). Hence, I instead seek to develop a framework in which we can objectively assess the optimality (or lack thereof) of propulsion in existing biological systems and, more importantly, arrive at optimal design strategies that reflect the different set of design constraints faced by the engineer as opposed to biological organisms.

Section 2 adds precision to the concept of optimal vortex formation by the analysis of vortex rings generated mechanically in the laboratory. The process leading to optimality is described heuristically and in analytical models. Section 3 presents evidence of optimal vortex formation in biological propulsion, beginning with cases explicitly seeking such optimality and finishing with examples having indirect or anecdotal support. Much of this section reexamines data that have been previously studied by using the concept of a dimensionless Strouhal frequency to describe the kinematics of locomotion. The primary aim here is to show that a direct quantification of the vortex formation itself can not only lead to the same conclusions previously deduced based on Strouhal frequency arguments, but can also indicate subtle differences in propulsion schemes that are often hidden by the Strouhal frequency.

Finally, Section 4 outlines some fundamental yet unanswered questions regarding the role of optimal vortex formation in biological propulsion. Wherever possible, I suggest the type of seminal experiment, analysis, or computation that could provide conclusive answers. Ultimately, this section is successful if it stimulates the reader to pursue one or more of the many remaining questions regarding the role of vortex dynamics in biological propulsion. Some of these questions are stated explicitly within the review, whereas others are intimated by gaps in the existing data. In either case, many uncertainties remain in the study of biological propulsion. The concept of optimal vortex formation can provide a new fluid mechanical basis for ongoing and future research in this area.

\section{OPTIMAL VORTEX FORMATION}

\subsection{Definitions}

To define optimal vortex formation, we must first parameterize the vortex formation process. The key concept in this regard is a dimensionless vortex formation time $\hat{T}$. It is defined based on the instantaneous vortex strength $\Gamma$ (i.e., circulation, $\mathrm{m}^{2} \mathrm{~s}^{-1}$ ), as well as the strength $\Delta U$ (i.e., 
$U-U_{\text {ambient }}, \mathrm{m} \mathrm{s}^{-1}$ ) and characteristic length scale $D(\mathrm{~m})$ of the shear layer feeding the vortex:

$$
\hat{T}=\frac{C \Gamma}{D \Delta U} \text {. }
$$

The constant factor $C$ depends on the physical configuration of the vortex generator. More specifically, $C$ is given by the inverse of the dimensionless vorticity flux $d \hat{\Gamma} / d \hat{T}$ from a given vortex generator configuration.

Historically, vortex ring formation has been most commonly studied using a piston-cylinder apparatus. In this configuration, a boundary layer of vorticity forms on the inner cylinder wall as the piston moves downstream inside the cylinder. At the downstream edge of the cylinder, the boundary layer separates and rolls up into a vortex ring that propagates away from the vortex generator via self-induction. The dimensionless vorticity flux $d \hat{\Gamma} / d \hat{T}$ from this configuration is approximately 1/2 (Didden 1979); therefore, the parameter $C$ in Equation 1 is equal to 2 in this case.

We can approximate the circulation $\Gamma$ generated by the piston-cylinder apparatus using the slug model (Didden 1979, Lim \& Nickels 1995), which assumes that within the boundary layer of thickness $\delta \ll D$, the wall-normal velocity component $v$ is much smaller than the streamwise component $u$. Also, the flow outside the boundary layer is assumed to be spatially uniform and equal to a constant $U$, the jet velocity. Under these conditions, we may write the circulation as

$$
\Gamma \approx \int_{0}^{T} \int_{D / 2-\delta}^{D / 2} u \omega_{\phi} d y d t \approx \int_{0}^{T} \int_{D / 2-\delta}^{D / 2} u\left(-\frac{d u}{d y}\right) d y d t=\int_{0}^{T}\left[-\frac{u^{2}}{2}\right]_{D / 2-\delta}^{D / 2} d t=\frac{1}{2} U^{2} T,
$$

where $\omega_{\phi}$ is the azimuthal component of vorticity, $y$ is the wall-normal direction $(-D / 2<y<D / 2$ across the cylinder exit plane), and $T$ is the duration of the vorticity flux from the piston-cylinder apparatus.

We can combine the result from Equation 2 with the known piston-cylinder shear layer strength $\left(\Delta U=U-U_{\text {ambient }}=U\right)$ and length scale (taken as the cylinder exit diameter $\left.D\right)$ to arrive at a dimensionless vortex formation timescale based on the physical parameters of the vortex generator:

$$
\hat{T}=\frac{2\left(\frac{1}{2} U^{2} T\right)}{D U}=\frac{U T}{D} .
$$

For consistency in the following discussion, we use $t$ to denote the advancing dimensional time during vortex formation and $T$ to denote the final dimensional time at the end.

\subsection{A Limiting Process}

The study of vortex ring formation has a rich history, much of which is well described in reviews by Shariff \& Leonard (1992) and Lim \& Nickels (1995); therefore I do not revisit it in detail here. However, it is noteworthy that, up to and including these reviews, this process was almost entirely studied for short dimensionless vortex formation times $\hat{T}<4$ (e.g., Auerbach 1991; Baird et al. 1977; Bernal \& Kwon 1989; Didden 1979; Glezer \& Coles 1990; Maxworthy 1972, 1977; Sallet \& Widmayer 1974; Schatzle 1987; Weigand \& Gharib 1994). Gharib et al. (1998) examined the vortex formation process for longer times, $\hat{T}>4$, and observed a robust limit on the maximum growth of vortex rings formed using a piston-cylinder apparatus. As illustrated in Figure 1, the forming vortex ring accepts the vorticity flux from the piston-cylinder apparatus until a dimensionless formation time of $\hat{T} \approx 4$ is reached. Beyond this time, additional vorticity flux is rejected by the vortex ring and instead forms secondary vortices akin to a Kelvin-Helmholtz instability. Gharib et al. (1998) termed this process vortex ring pinch-off. This process is not to be confused with the

Vortex ring pinch-off: the process whereby a forming vortex ring is no longer able to entrain additional vorticity from a vortex generator 

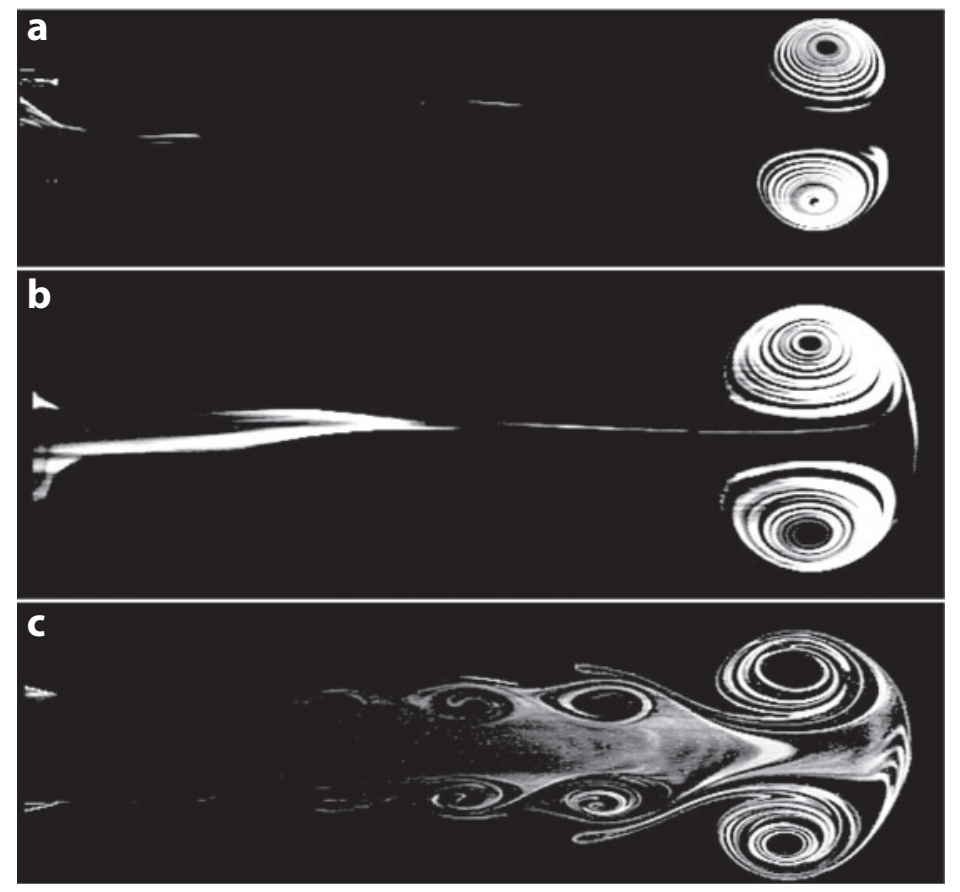

Figure 1

Visualization of vortex rings at downstream position $X / D \approx 9$ for $(a) \hat{T}=2,(b) \hat{T}=3.8$, and $(c) \hat{T}=14.5$. Figure taken from Gharib et al. 1998.

physical separation between the vortex ring and the trailing shear layer, which may occur later or not at all.

We can understand the existence of a limiting dimensionless timescale for vortex ring formation in terms of the energetics of the formation process. As described by Kelvin (1880) and Benjamin (1976), a steady, symmetry-axis-touching vortex ring possesses maximum energy relative to the equivalent rearrangements of its vorticity that maintain the same total impulse. Therefore, a forming vortex ring can only accept additional vorticity (e.g., from a trailing shear layer) and subsequently relax to a new configuration if the impulse-normalized energy of the new configuration is greater than the impulse-normalized energy of an alternative vortex ring/shear layer configuration in which the additional vorticity is not accepted by the vortex ring. Pozrikidis (1986) observed examples of the latter configuration using numerical simulations in which steady vortex rings were locally perturbed. Figure $\mathbf{1} \boldsymbol{c}$ also shows an example of a vortex ring/shear layer con-

Kelvin-Benjamin variational principle: states that a steady axis-touching vortex ring possesses maximum energy with respect to alternative arrangements of the vorticity with the same total impulse figuration that results when the requirement of the Kelvin-Benjamin variational principle is not satisfied and vortex ring pinch-off occurs.

By using the slug model to quantify the energy of the shear layer delivered by the piston-cylinder apparatus and by using the Norbury-Fraenkel family of vortex rings (Fraenkel 1972, Norbury 1973) to classify the experimentally generated ones, Gharib et al. (1998) quantitatively predicted the observed limiting timescale for vortex ring formation. Whereas the normalized energy of the leading vortex appears relatively constant in the model, the normalized energy of the shear layer feeding the vortex ring decreases monotonically in dimensionless time. The crossover point at which $\hat{E}_{\text {shear layer }}<\hat{E}_{\text {vortex ring }}$ occurs near $\hat{T} \approx 4$, in agreement with experiments. Subsequent models based on flow kinematics (Linden \& Turner 2001, Shusser \& Gharib 2000), more complex 
vortex ring families (Kaplanski \& Rudi 2005, Mohseni \& Gharib 1998), and statistical physics (Mohseni 2001) predict the existence of this process that limits the maximum growth of vortex rings during formation.

Irrespective of these particular models, Equation 1 gives some physical intuition as to how the various dimensional parameters relevant to vortex ring formation affect the limiting formation process. For example, stronger vortices (i.e., larger circulation $\Gamma$ ) tend to be more energetic (Lamb 1932) and therefore advance dimensionless vortex formation time $\hat{T}$ more rapidly. Conversely, the vortex formation process can be extended by either a stronger shear layer (i.e., larger $\Delta U$ ) or a larger shear layer (i.e., larger $D$ ) feeding the vortex ring, both of which tend to delay the advancement of the dimensionless vortex formation time. The constant $C$ in Equation 1, which depends on the dimensionless vorticity flux from the vortex generator, dictates the specific numerical value of $\hat{T}$ at which vortex ring growth is limited. For the piston-cylinder arrangement, the limiting value of $\hat{T}$, also known as the vortex formation number, is approximately 4 . As shown below, other vortex formation numbers exist in vortex ring generator configurations in which $C$ presumably takes on different values.

\subsection{A Constrained Optimization}

Given that vortex ring growth is limited by the Kelvin-Benjamin variational principle, one may ask whether there are beneficial properties of vortex ring formation that scale with increasing vortex size. If so, then to achieve those benefits maximally, we wish to maximize the size of each generated vortex ring given the aforementioned constraint imposed by the dimensionless vortex formation time $\hat{T}$. This is the essence of optimal vortex formation: The quantity that is optimized per se is that which scales with increasing vortex ring size.

In a seminal work, Krueger (2001) experimentally deduced some of the properties of vortex ring formation that scale with increasing vortex size and are therefore potential targets for optimal vortex formation. He determined that the normalized time-averaged thrust per jet pulse, $\hat{F}=\bar{F} / \rho A U^{2}$ (where $\rho$ is the fluid density, $A$ is the cylinder exit area, and the overbar denotes a time average), was one such parameter, reaching its maximum value just before the limiting dimensionless vortex formation time $\hat{T} \approx 4$ (Figure 2 ). For a constant cylinder exit velocity $U$, the

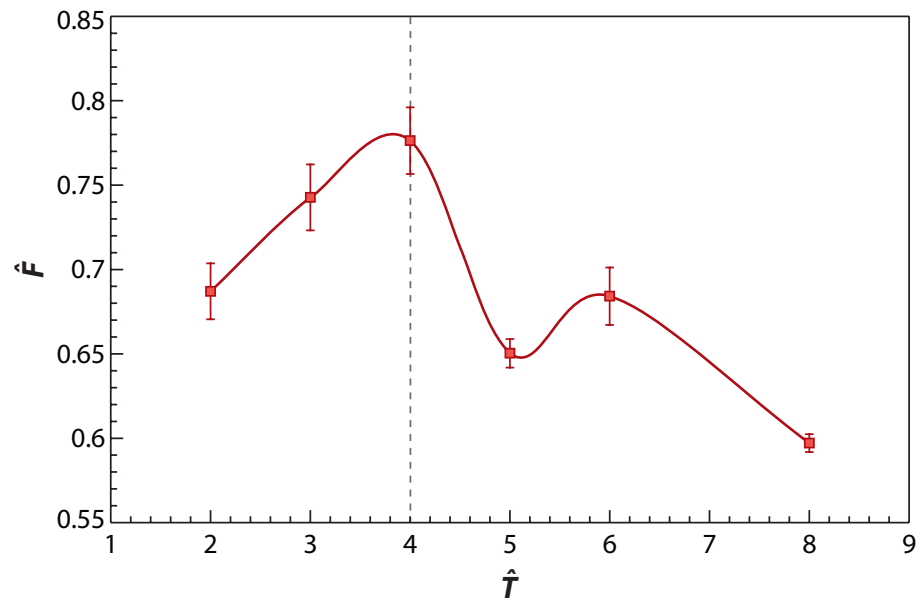

Figure 2
Vortex formation number: the vortex formation time at which the total circulation supplied by the vortex generator is equal to the pinched-off vortex ring circulation

Normalized average thrust per pulse measurements. Figure taken from Krueger \& Gharib 2003. 
denominator of $\hat{F}$ can be multiplied by $U$, resulting in a parameter that is effectively a measure of the energetic efficiency of propulsion via vortex formation. In other words, by maximizing the size of the vortex rings formed during propulsion, the efficiency of momentum transport is also optimized.

Physically, the benefit of vortex ring formation for propulsion arises because of the entrainment of ambient fluid by the forming vortex ring (Auerbach 1991, Dabiri \& Gharib 2004b, Olcay \& Krueger 2007, Shadden et al. 2007), as well as the added mass of nonentrained fluid surrounding the vortex that must be accelerated with the vortex ring (Baird et al. 1977, Krueger \& Gharib 2003, Weihs 1977). This latter effect is mathematically equivalent to the added mass carried with a solid body in a potential flow, and it can be computed in terms of the velocity potential $\phi$ of the flow outside the vortex (Dabiri 2006). The propulsive reaction force experienced by the vortex generator is proportional to the sum of the shear layer source fluid, entrained fluid, and the added mass of the fluid surrounding the vortex ring (Dabiri 2005, Krueger 2001).

\subsection{Is the Vortex Ring Formation Number Universal?}

The existence of a constrained optimization during vortex formation as elucidated above immediately leads one to question whether the constraint (i.e., the limiting process during vortex formation) can be manipulated for increased propulsive gains. A related question is whether the specific value of the vortex formation number (e.g., $\hat{T} \approx 4$ for the piston-cylinder apparatus) is generic to all vortex shedding. I address these issues in turn below. Before describing those results, however, I distinguish between methods that affect the vortex ring formation process in dimensional time but are still constrained by a limiting dimensionless vortex formation time $\hat{T} \approx 4$ and methods that actually change the limiting vortex formation time from $\hat{T} \approx 4$. Either method can potentially benefit propulsion, but the latter has the potential to do so to a greater extent because it directly affects the physical constraint on optimal vortex formation. In cases below in which the constant parameter $C$ in Equation 1 is manipulated via the dimensionless vorticity flux, the limiting dimensionless

vortex formation time $\hat{T}$ can be changed from 4 . However, if the proposed methods only affect the parameters $\Gamma, \Delta U$, and $D$ in Equation 1 independent of the parameter $C$, the dimensionless pinch-off need not be changed from its usual value $\hat{T} \approx 4$ despite its delay in dimensional time.

2.4.1. Piston-cylinder studies. Rosenfeld et al. (1998) studied the vortex ring formation process using numerical simulations of a piston-cylinder apparatus, following the vortex ring experiments of Gharib et al. (1998) and Johari et al. (1996). They showed that the vortex formation number could be reduced by as much as $75 \%$ or increased by $35 \%$ by manipulating the temporal and spatial profiles of the cylinder exit velocity. Mohseni et al. (2001) demonstrated additional mechanisms to delay vortex ring pinch-off by using a nonconservative forcing to numerically simulate the vortex ring formation process without explicitly modeling the piston-cylinder boundary conditions. They suggested two mechanisms capable of extending the vortex ring growth process observed in pistoncylinder experiments. The first method was to accelerate the trailing shear layer relative to the forming vortex ring so that the shear layer energy was sufficient to be accepted by the forming vortex ring and to consequently achieve a new steady vortex ring configuration. This method is consistent with Shusser \& Gharib's (2000) proposed kinematic model of vortex ring pinch-off, which describes the process in terms of a physical separation between the forming vortex ring and the trailing shear layer. As the circulation of the leading vortex ring increases during formation, the vortex ring accelerates downstream under increasing self-induction. It is therefore necessary (but not sufficient) for the trailing shear layer to also be accelerated in order to remain sufficiently close to the forming vortex to continue feeding it with vorticity. 
Mohseni et al.'s (2001) second method to delay the limiting vortex formation time $\hat{T}$ was to increase the radial extent of the trailing shear layer. Their simulations suggested that this would delay the onset of the symmetry-axis-touching state of the shear layer, which they correlated with vortex ring pinch-off.

A variety of piston-cylinder configurations have also been studied experimentally in recent years to determine the generality of the limiting vortex formation time $\hat{T} \approx 4$. Dabiri \& Gharib (2004a) demonstrated modest pinch-off delays of approximately $10 \%$ in the limiting vortex formation time by immersing a piston-cylinder apparatus in a uniform bulk counterflow. Krueger et al. (2006) demonstrated the opposite effect, a reduction in the vortex formation number in the case of uniform bulk coflow around a piston-cylinder apparatus. For coflow velocity ratios $U_{\text {coflow }} / U_{\text {jet }}>0.5$, vortex ring pinch-off occurred almost immediately following the initiation of the vortex ring's formation. Finally, by temporally varying the cylinder exit diameter during formation, Dabiri \& Gharib (2005a) showed that vortex ring pinch-off could be delayed up to $\hat{T} \approx 8$.

In summary, numerical and experimental studies demonstrate the possibility of manipulating the limiting dimensionless vortex formation time from $\hat{T} \approx 4$. However, it does not appear possible to eliminate the limiting process altogether. This conclusion has stimulated the search for optimal vortex formation in other canonical flows unrelated to the piston-cylinder apparatus (see Section 2.4.2). During this search, one must exercise care in defining the dimensionless time $\hat{T}$ so that differences in the vortex generator configuration alone (i.e., independent of the fluid dynamics) are not sufficient to produce differences in the observed limiting vortex formation time. For example, the studies of bulk counter- and coflow described above required the ambient flow to be accounted for in the dimensionless time as

$$
\begin{aligned}
& \hat{T}=\frac{\left(U_{\text {jet }}-U_{\text {counterflow }}\right) T}{D} \quad \text { (counterflow), } \\
& \hat{T}=\frac{\left(U_{\text {jet }}+U_{\text {coffow }}\right) T}{D} \quad \text { (coflow). }
\end{aligned}
$$

These definitions follow directly from the general form in Equation 1, with the ambient flow contributing a factor $-\frac{1}{2} U_{\text {coffow/counterflow }}^{2} T$ to the circulation $\Gamma$ and a factor $\mp U_{\text {coflow/counterflow }}$ to the shear $\Delta U$. If the coflow/counterflow term is neglected in the definition of the dimensionless time, erroneous conclusions regarding the ambient flow's effect on the limiting vortex formation time $\hat{T}$ can result. Similarly, in a study involving a time-dependent cylinder exit diameter, Dabiri \& Gharib (2005a) integrated the instantaneous shear layer length scale $D(t)$ in time to derive a dynamic formation time that describes the vortex formation process:

$$
\hat{T}=\int_{0}^{T} \frac{U(t)}{D(t)} d t .
$$

If using a time average of the length scale $D$ in Equation 3 instead of the time integration (Equation 5), one could draw erroneous conclusions regarding the physical relevance of the limiting dimensionless vortex formation time (Dabiri \& Gharib 2005b).

2.4.2. Optimal vortex formation in other vortex-shedding configurations. Before making the case for the existence of optimal vortex formation in biological propulsion, let us examine other canonical flows that strongly suggest the occurrence of optimal vortex formation. The common feature among these flows and the piston-cylinder flow is the generation of vortex rings or derivatives thereof (e.g., vortex loops and chains) in the wake. Jeon \& Gharib (2004) studied the flow generated by a starting circular cylinder and by a steadily oscillating circular cylinder, aiming to identify the signature of optimal vortex formation in the wake. They noted that the 
shedding of the first vortex pair from the starting cylinder occurred at the same dimensionless time $\hat{T} \approx 4$ as observed in previous studies of vortex rings generated by a piston-cylinder apparatus. Furthermore, the transition from two single vortices shed per cycle to two pairs of shed vortices in the wake of the steadily oscillating circular cylinder (Ongoren \& Rockwell 1988, Williamson \& Roshko 1988) appeared to also correlate with the limiting timescale for vortex ring formation.

Milano \& Gharib (2005) experimentally studied the vortex wake of an oscillating flat plate and applied a genetic algorithm to optimize the time-averaged thrust generated by the plate. Similar to the conclusions of Krueger and colleagues (Krueger 2001, Krueger \& Gharib 2003), they observed that optimal force production occurred for flat-plate oscillations corresponding to a dimensionless vortex formation time $\hat{T} \approx 4$. The definition of the dimensionless time was modified as in Dabiri \& Gharib (2005a) (Equation 5) to account for the time-varying projected area of the flat plate as it pitched about the leading edge.

Ringuette et al. (2007) studied the wake of an accelerating low-aspect-ratio flat plate and observed pinch-off of the vortex pair behind the plate at a dimensionless time $\hat{T} \approx 4$. Interestingly, this time also corresponded to that of the drag coefficient on the plate relaxed to its quasi-steady value.

In each of these canonical flows, pinch-off is understood in terms of the three-dimensional flow around the object. Thus, although is it customary to consider dipole shedding from cylinders and flat plates, the connection with optimal vortex formation requires a view toward the threedimensional vortex ring wake structure. That the vortex rings in the wake are not circular or isolated as in the piston-cylinder studies does not seem to preclude the existence of an optimal vortex formation process in these flows. It is therefore reasonable to consider that optimal vortex formation may be a general feature in flows with coherent, three-dimensional vortex shedding. Biological propulsion represents a substantial subset of such flows. To identify the occurrence of optimal vortex formation in biological propulsion, we must determine the proper extensions of the timescale given in Equation 1 to the propulsor kinematics found in biological systems. We pursue this goal in the following section, seeking evidence of optimal vortex formation in classes of biological propulsion for which vortex shedding of various kinds is commonplace.

\section{EVIDENCE FOR OPTIMAL VORTEX FORMATION IN BIOLOGICAL PROPULSION}

\subsection{Direct Studies of Optimal Vortex Formation}

The qualitative similarity between some biological flows and the vortex rings formed in pistoncylinder experiments motivated a series of studies aiming to identify optimal vortex formation in biological propulsion. The following sections summarize the results of those investigations.

3.1.1. Blood flow in the cardiac left ventricle. The left heart is responsible for the collection of oxygenated blood from the pulmonary circulation and the subsequent distribution of that blood throughout the body. A key process within the left heart is the transfer of blood from the left atrium to the left ventricle via a starting jet that forms during early diastole (relaxation) of the left heart. During initiation of this starting jet, a vortex forms downstream from the mitral valve that separates the left atrium and left ventricle (Domenichini et al. 2005, Kim et al. 1995). This process of blood transport correlates with the overall health of the heart (e.g., Kilner et al. 2000); however, existing metrics that quantify cardiac health do not specifically account for the vortex formation process. Gharib et al. (2006) used the optimal vortex formation concept to quantify vortex formation in the left heart of patients measured using echocardiography. In a blind study 
a

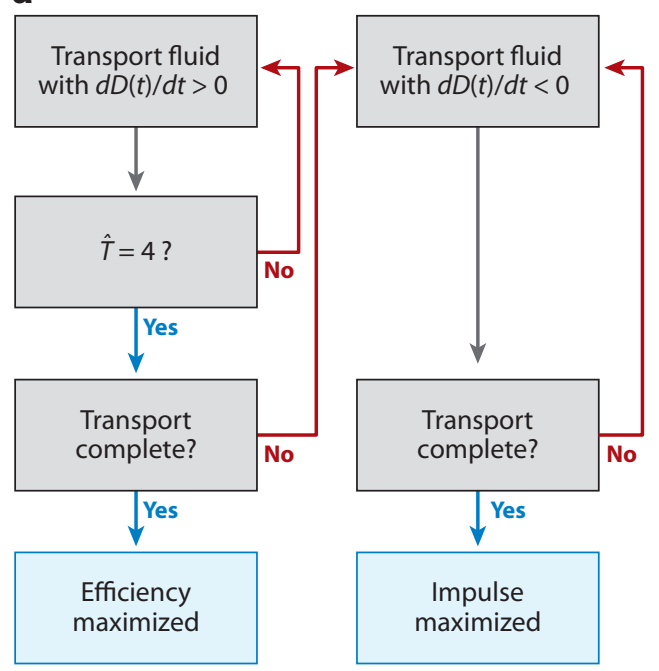

b

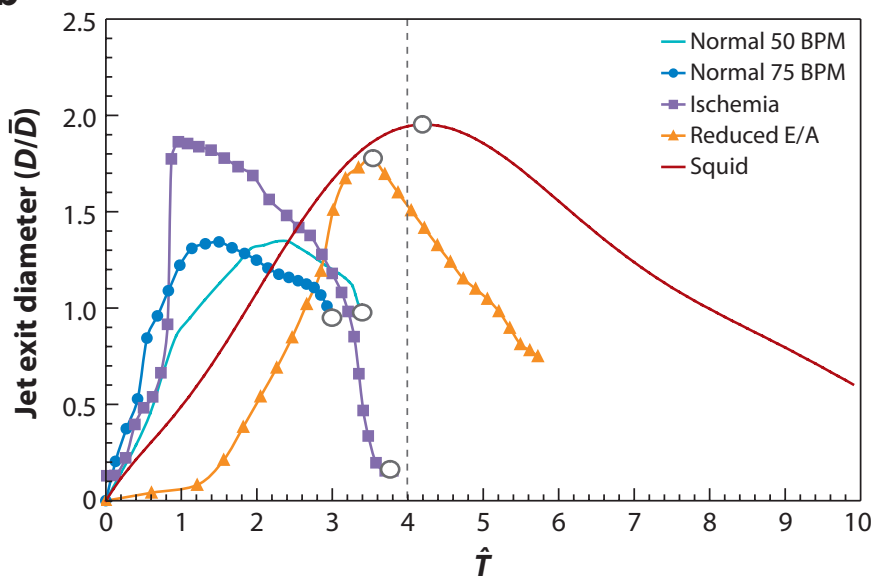

Figure 3

(a) Optimal vortex formation protocols for maximizing efficiency or impulse. (b) Measurements of mitral valve exit diameter $D$ versus $\hat{T}$ for various left ventricle conditions and measurements of funnel diameter $D$ versus $\hat{T}$ for squid jet propulsion. Initial funnel diameter is subtracted from the measurements for normalization. Open circles indicate points of correlation with optimal vortex formation time based on panel $a$. E/A is the ratio of blood-flow velocities during early diastolic filling and atrial contraction, respectively. Panel $b$ adapted from Dabiri \& Gharib 2005b.

of 120 normal volunteers, they observed that vortex formation in the left ventricle occurred in a dimensionless time $\hat{T} \approx 4$, similar to the result of previous laboratory studies of vortex ring formation. Furthermore, patients with abnormal left heart function (i.e., dilated cardiomyopathy) exhibited significantly lower vortex formation times, indicating suboptimal fluid transport.

Gharib et al. (2006) used time-averaged values of the mitral valve exit diameter owing to the limited temporal resolution of the echocardiogram measurements. Dabiri \& Gharib (2005b) hypothesized that the temporal variations in the characteristic length scale of the shear layer $D(t)$ could provide additional information regarding the efficacy of fluid transport. They proposed a protocol based on the limiting dimensionless vortex formation time $\hat{T}$ and the time-varying length scale $D(t)$ that could maximize either efficiency or impulse (Figure $3 \boldsymbol{a}$ ). There are two basic tenets of the protocol: $(a)$ Vortex ring impulse is directly proportional to the characteristic length scale $D$, and $(b)$ quasi-steady jet impulse from a constant mass flux source is inversely proportional to $D$. Therefore, during jet initiation when vortex formation occurs, $D$ should be increased in time to take advantage of the first tenet. If fluid transport is terminated at dimensionless time $\hat{T} \approx 4$, the sections above show that efficiency is maximized. However, to maximize impulse, one needs to extend the fluid transport beyond $\hat{T} \approx 4$ while decreasing the length scale $D$, per the second tenet. Here, the trailing jet behind the pinched-off vortex ring provides the primary source for fluid impulse.

Dabiri \& Gharib (2005b) analyzed measurements of transmitral blood flow for patients with normal and pathological cardiac function, and they observed that the proposed protocol in Figure $3 \boldsymbol{a}$ was manifested in each case studied. In particular, the most severe pathology (i.e., reduced E/A) exhibited a temporal trend of $D$ versus $\hat{T}$ that indicated a dramatic transition from efficient propulsion to a propulsion that maximizes impulse (Figure $3 \boldsymbol{b}$ ). A possible explanation for this transition is that for cases of severe dysfunction, efficient blood transport must be sacrificed to merely achieve the transport of mass and momentum required for the continued 
operation of the left heart. Kilner et al. (2000) and Kheradvar et al. (2007) have confirmed that a baseline fluid impulse is required in the transmitral blood flow to facilitate proper recoil and filling of the left ventricle.

3.1.2. Squid jet propulsion. Squid jet propulsion shows an interesting parallel with the cardiac functions described in the previous section. During low-speed swimming, squids use lateral fins for propulsion instead of the jet formed by contractions of the mantle. However, at higher speeds and when escaping predators, they use the jet-propelled mode of locomotion (Anderson \& DeMont 2000, Bartol et al. 2001, O'Dor 1988). It is therefore reasonable to presume that efficiency is not always a squid's primary objective for jet-propelled swimming because efficiency becomes secondary to survival during escape swimming. Studies support this conclusion, showing that the cost of jetpropelled swimming is high for some squids and that the jets produced by adult squid can persist much longer than $\hat{T} \approx 4$ (Anderson \& Grosenbaugh 2005). Dabiri \& Gharib (2005b) used measurements (I.K. Bartol, personal communication) of the jet velocity and funnel (i.e., jet exit) diameter to compute the temporal trend of $D$ versus $\hat{T}$. They found that the squid swimming kinematics during jet propulsion were similar to the proposed protocol for impulse maximization as opposed to efficiency maximization (Figure $3 b$ ). From this perspective, a squid's survival and the human heart appear to depend on similar optimal vortex formation strategies. As the data studied by Dabiri \& Gharib (2005b) were a limited sample, more study is required to firmly establish these conclusions.

Bartol et al. (I.K. Bartol, P.S. Krueger, J.T. Thompson \& W.J. Stewart, manuscript submitted) are headed in that direction, as their studies of hatchling, juvenile, and adult squids are consistent with the bimodal propulsion paradigm of Figure $3 \boldsymbol{a}$. Whereas the transition from maximum efficiency to maximum impulse occurs as a disease pathology in the left heart, this transition occurs in squid as part of ontogenetic development from hatchling to adult, concomitant with lateral fin development.

3.1.3. Jellyfish swimming. Although jellyfish swimming is generally referred to as jet propulsion, only a relatively small subset of jellyfish creates a single vortex ring per swimming cycle in a manner similar to squid jet propulsion. Dabiri et al. (2006) studied one species that does create single vortex rings, Nemopsis bachei, using passive dye markers to observe the wake structure. Based on the swimming kinematics of the body and related models, they determined that the fluid efflux persisted up to $\hat{T} \approx 8$. However, vortex ring pinch-off did not occur at all. They therefore concluded that the time-varying motion of the velum (i.e., jet exit) resulted in shear layer kinematics that delayed the limiting vortex formation process to at least $\hat{T} \approx 8$. This factor of two increase in the limiting dimension vortex formation time is consistent with the laboratory experiments of Dabiri \& Gharib (2005a), who used a piston-cylinder apparatus to study the effect of similar time-dependent shear layer kinematics $D(t)$. Notably, Bartol et al.'s (I.K. Bartol, P.S. Krueger, J.T. Thompson \& W.J. Stewart, manuscript submitted) studies of squid swimming also indicate vortex formation numbers up to $\hat{T} \approx 8$.

3.1.4. Other studies. Linden \& Turner (2004) conducted a wide-ranging study of various modes of aquatic locomotion, seeking to identify optimal vortex formation based on existing measurements in the literature. The results were mixed, with evidence of optimal vortex formation in juvenile squid, ambiguous results for jellyfish and salps, and negative results for adult squid (but see Section 3.1.2 above). They observed possible indications of optimal vortex formation for various modes of fish swimming, but those conclusions relied on an assumed relationship between the dimensionless vortex formation time and the Strouhal frequency of the fish's swimming kinematics. We revisit that relationship in the following section. 
These and the preceding examples emphasize that identifying optimal vortex formation in biological propulsion systems requires more than a search for a specific vortex formation number (e.g., $\hat{T} \approx 4$ ) or an assumption that energetic efficiency is the target of optimization (cf. Figure $3 a$ ). Nonetheless, because optimal vortex formation is intimated across the diverse examples described here, there is confidence that optimal vortex formation does indeed remain to be discovered in many other biological systems as well.

\subsection{Indirect Studies of Optimal Vortex Formation: Strouhal Frequency Constraints}

Animal locomotion kinematics are commonly described using the dimensionless Strouhal frequency $\mathrm{St}$, its definition based on the dimensional frequency $f_{s}$ of the appendage stroke cycle, the peak-to-peak amplitude of the appendage stroke $D_{s}$, and the forward velocity $U_{a}$ of the animal:

$$
\mathrm{St}=f_{s} D_{s} / U_{a} \text {. }
$$

This parameter is tightly constrained for a broad range of locomotion modes in water and air, including fish and mammal swimming and bird and bat flight (Taylor et al. 2003). Explanations for this constraint given in the literature include selective pressure for propulsive efficiency, the resonant frequency of the musculature, and stability modes of the time-averaged wake (e.g., Alborn et al. 2006; Fish \& Lauder 2006, and references therein; Triantafyllou et al. 2000). Let us now consider the possibility that the Strouhal frequency constraint is primarily a consequence of the process of optimal vortex formation.

Comparison of the Strouhal frequency definition in Equation 6 to the dimensionless vortex formation-time definition in Equation 3 suggests an inverse relationship between the two parameters. However, there is a not a one-to-one correspondence between the independent variables in the dimensionless vortex formation time and the Strouhal frequency. For example, the duration of vortex formation $T$ is only equal to the inverse of the stroke frequency $f_{s}$ if a single vortex ring is shed by the appendage per stroke cycle. In certain Strouhal frequency regimes, two vortex rings are shed per cycle (Buchholz \& Smits 2006, Dong et al. 2006); hence, $T=1 /\left(2 f_{s}\right)$ in these cases instead of $1 / f_{s}$. Also, the characteristic length scale $D$ of the shear layer feeding the vortex ring is a function of both the span and chord lengths of the appendage, whereas the Strouhal frequency is only dependent on the excursion of the tip of the appendage $D_{s}$. Finally, whereas the dimensionless vortex formation time depends on the strength of the shear layer $\Delta U$ and its advective velocity $U_{a d v}$, the Strouhal frequency is a function of the forward velocity of the animal, which may be different from the shear layer advective velocity.

To achieve a quantitative relationship between the Strouhal frequency and the dimensionless vortex formation time, let us instead begin with the more general form of Equation 3 given in Equation 1. In addition, we consider two simplified models of animal locomotion (Figure 4) to illustrate the application of these ideas. The first example is akin to the caudal fin locomotion of a fish: A flat plate with chord $c$ and span $s$ is oscillated through an angle $2 \alpha$ while the animal moves forward at speed $U_{a}$. The second example is similar to the first except that the orientation of the flapping is normal to the animal's forward motion, akin to bird flight. For both cases, our goal is to model the parameters $\Gamma, \Delta U$, and $D$ in the dimensionless vortex formation-time definition (Equation 1) to deduce the implicit connection with Strouhal frequency.

As an aside, if the parameters in Equation 1 are available from measurements or numerical simulations of the propulsion system, then studies using the optimal vortex formation time (Equation 1) can proceed directly. The models below are developed for the sake of those who do 
a

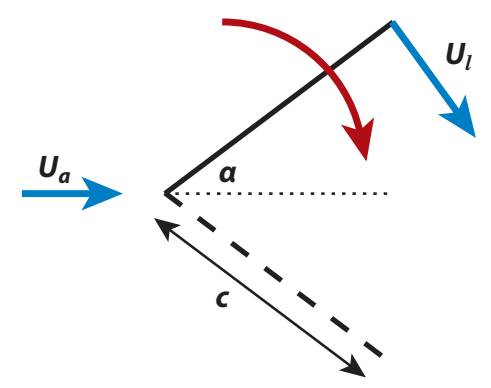

b

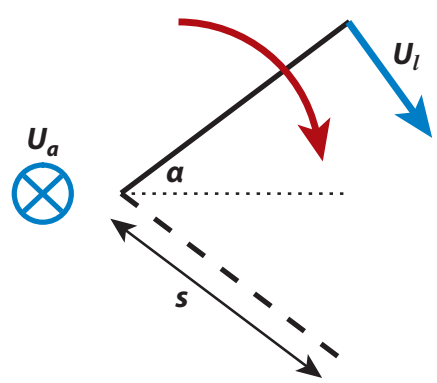

Figure 4

Simplified models of animal locomotion in fluids: (a) caudal fin and $(b)$ bird wing.

not have direct access to the governing physical parameters but do have basic knowledge of the appendage geometry and kinematics.

Because the shear layer that feeds the vortex wake is shed from both the chordwise and spanwise edges of the appendage, we take the characteristic shear layer length scale $D$ to be $4 A / P$, where $A$ is the projected area of appendage in the direction of its motion and $P$ is the perimeter of the appendage. For the piston-cylinder apparatus, an equivalent calculation based on the area of the cylinder exit and its circumference results in the length scale identified previously, i.e., $\frac{4\left(\pi D^{2} / 4\right)}{\pi D}=D$. The strength of the shear layer $\Delta U$ is given by the difference between the local velocity $U_{l}$ at the chordwise and spanwise edges of the appendage and the component of the ambient velocity $U_{a}$ in the direction of appendage motion (see Figure 4):

$$
\begin{aligned}
& \Delta U=\frac{1}{2}\left[2 \pi f c \pm U_{a} \sin \alpha(t)\right] \quad \text { (caudal fin), } \\
& \Delta U=\frac{1}{2}(2 \pi f s) \quad \text { (bird wing), }
\end{aligned}
$$

where the factor of $1 / 2$ reflects the linearly decreasing velocity along the chord of the model caudal fin and along the span of the model bird wing. Because the motion of the model bird wing is normal to the direction of animal motion, no component of $U_{a}$ contributes to the strength of the shear layer feeding the vortex wake. In reality, the wing's small angle of attack invalidates this assumption, and the reader can deduce how to incorporate that effect from the problem's geometry if desired. The current model is sufficient for what follows.

It remains to develop a model for the circulation of the wake vortex ring. As mentioned above, the circulation should ideally be measured directly in experiments or computed numerically. In the absence of these options, one can apply the boundary-layer model in Equation 2, with the assumption that the vorticity flux $d \Gamma / d t$ from the appendage is advected into the wake vortex at a speed $U_{a d v}$ over a duration $T$ :

$$
\Gamma \approx U_{a d v} \Delta U T \text {. }
$$

Substituting these models for $D, \Delta U$, and $\Gamma$ into Equation 1 gives

$$
\hat{T} \approx \frac{C U_{a d v} \Delta U}{D \Delta U f_{s} N}=\frac{C U_{a d v}}{D f_{s} N},
$$

where $N$ is the number of vortex rings shed per stroke cycle. By examining Equation 9, we see that for $\hat{T}=1 / \mathrm{St}$, we must also have, e.g., $U_{a d v}=U_{a} / 2, D=D_{s}, C=2$, and $N=1$. In cases that satisfy these assumptions, we can interpret the previously identified constraint on 
Strouhal frequency during locomotion in air and water to reflect a constraint on the dimensionless vortex formation time. Indeed, the range of Strouhal frequencies observed in biological propulsion $(0.2<$ St $<0.4$; Taylor et al. 2003) corresponds to the range of limiting dimensionless time for optimal vortex formation. However, unlike the Strouhal frequency, the optimal vortex formation time can be predicted based on physical arguments directly related to the energetics of vortex formation. The dimensionless vortex formation time also can be measured for single stroke cycles. Therefore, locomotive modes in which a steady cruising velocity $U_{a}$ is undefined (e.g., insect hovering and bird and fish maneuvering) can be treated using the dimensionless vortex formation time, whereas the Strouhal frequency is undefined. In using the dimensionless vortex formation time, we remind the reader that time-dependent kinematics may require time integration of the vortex formation time as in Equation 5.

In cases that do not satisfy the assumptions $U_{a d v}=U_{a} / 2, D=D_{s}, C=2$, and $N=1$ (or, more generally, $\hat{T}=1 / \mathrm{St}$ ), the dimensionless vortex formation time provides additional parametric dependencies that can be used to classify and evaluate various modes of locomotion that appear identical in a Strouhal frequency classification. The shortcomings related to such misclassifications based on the Strouhal frequency have been illustrated by measurements demonstrating circumstances in which the Strouhal frequency alone is insufficient to indicate optimal propulsion (Bandyopadhyay \& Beal 2007).

\section{OUTLOOK}

\subsection{Analytical Directions}

Relative to the study of vortex dynamics or even of biological propulsion, the concept of optimal vortex formation is far from maturity. Most lacking is a rigorous extension of the Kelvin-Benjamin variational principle to configurations of vorticity that do not form symmetry-axis-touching vortex rings. The existing data suggest that the concept of optimal vortex formation does indeed extend to these more complex cases; however, as shown above, changes in vortex generator geometry affect the specific value of dimensionless time $\hat{T}$ at which vortex growth is limited. The ability to predict the optimal vortex formation time for arbitrary vortex generator geometries will inform the search for its occurrence in measurements of biological propulsion systems.

\subsection{Experimental Directions}

No two biological propulsion systems function identically, even systems of the same species and age. We can also expect cycle-to-cycle variations in vortex formation within that same propulsor. To what extent is the optimal vortex formation process robust to these variations? Gharib et al.'s (1998) laboratory measurements indicate variations of up to $15 \%$ in the optimal dimensionless vortex formation time during their experiments. It is currently unknown how close to the nominal optimal vortex formation time a system must operate to maintain the associated performance benefits. The experiments of Krueger and colleagues (Krueger 2001, Krueger \& Gharib 2003) provide some indication for the piston-cylinder apparatus (e.g., Figure 2), but the generality of their measured trends is unknown. This question is particularly important with regard to the fitness of a population of similar propulsion systems. Diagnosis of left heart failure provides a poignant example in which natural variability must not be mistaken for suboptimal performance lest falsepositive indications of cardiac dysfunction arise. In the realm of bio-inspired propulsion, it is useful to know to what degree design objectives that compete with the achievement of the optimal vortex formation time can be accommodated, and to what degree that optimum can be maintained in the face of unknown design variables (e.g., ambient wind/water conditions around a vehicle). 


\section{EXTRACTING VORTICES FROM BIOLOGICAL FLOWS}

Recently developed Lagrangian methods of flow analysis can substantially aid the identification of vortex rings in experimentally measured biological flows. Haller (2001), Shadden et al. (2005, 2006), Green et al. (2007), and others have demonstrated that by computing the finite-time Lyapunov exponent field corresponding to the flow of interest, one can identify Lagrangian coherent structures-such as vortex rings-as ridges of local maxima in the finite-time Lyapunov exponent field. Lagrangian coherent structure methods are effective in flows with arbitrary unsteadiness, in which Eulerian identification methods based on the velocity and vorticity fields can be insufficient. This technique is also robust to local measurement errors (Haller 2002), making it especially useful in analyzing complex animal wake kinematics.

Few animals have been studied from a perspective aiming to quantify the occurrence of optimal vortex formation. A broad area of opportunity exists for experimentalists to revisit previous measurements and to conduct new ones to address this deficiency, especially with respect to the parameters needed to compute Equation 1.

\subsection{Computational Directions}

As mentioned above, the study of optimal vortex formation is inherently a three-dimensional fluid dynamic problem. Given current limitations in our ability to measure such flows experimentally, computational tools can provide valuable insight into the process of optimal vortex formation. Recent numerical work has confirmed expectations from experimental studies (e.g., Buchholz \& Smits 2006), suggesting the formation of well-defined vortex ring structures in the wakes of biological and biomimetic foils (Aono et al. 2008, Dong et al. 2006). These data sets may be ideal for investigating the process of optimal vortex formation because the parameters in Equation 1 can be identified without ambiguity. Furthermore, large parametric studies aimed at determining the functional dependence of the dimensionless vortex formation time on the propulsor design can be accomplished in silico with less expense than an equivalent experimental study. Mohseni et al. (2001) accomplished this type of study for the piston-cylinder apparatus, leading subsequently to more targeted experimental studies.

In summary, the concept of optimal vortex formation not only provides a unifying framework in which to understand biological propulsion, it also suggests an objective metric with which to design and evaluate engineered propulsion systems. To exploit the concept of optimal vortex formation for the greatest advantage to the engineer and biologist, we must improve our understanding of the governing physical principles of optimal vortex formation. I hope that the foregoing discussion provides a useful orientation and roadmap toward that goal.

\section{SUMMARY POINTS}

1. Studies of vortex ring formation indicate a limiting growth process dictated by the KelvinBenjamin variational principle.

2. Transport of mass, momentum, and energy by vortex rings can be optimized by maximizing vortex ring formation within the energetic constraint imposed by the KelvinBenjamin variational principle. 
3. Optimal vortex formation is suggested in more complex vortex systems, including cylinder and flat-plate cross-flow.

4. Biological systems (including the human heart and swimming and flying animals) exhibit the signatures of optimal vortex formation; however, the effect has only been measured definitively in a few cases.

5. By understanding optimal vortex formation as it occurs in biological propulsion, engineers can gain inspiration for the design of more effective propulsion technologies.

6. Future analytical, experimental, and computational efforts should aim to determine the generality of optimal vortex formation for more complex vortex kinematics.

\section{DISCLOSURE STATEMENT}

The author is not aware of any biases that might be perceived as affecting the objectivity of this review.

\section{ACKNOWLEDGMENTS}

The author is indebted to Morteza Gharib for discussions and collaborations that motivated this review. The support of the National Science Foundation programs in Ocean Sciences, Ocean Technology, and Energy for Sustainability is gratefully noted, as is an Office of Naval Research Young Investigator Program award.

\section{LITERATURE CITED}

Alborn BK, Blake RW, Megill WM. 2006. Frequency tuning in animal locomotion. Zoology 109:43-53

Anderson EJ, DeMont ME. 2000. The mechanics of locomotion in the squid Loligo pealei: locomotory function and unsteady hydrodynamics of the jet and intramantle pressure. F. Exp. Biol. 203:2851-63

Anderson EJ, Grosenbaugh MA. 2005. Jet flow in steadily swimming adult squid. 7. Exp. Biol. 208:1125-46

Aono H, Liang F, Liu H. 2008. Near- and far-field aerodynamics in insect hovering flight: an integrated computational study. F. Exp. Biol. 211:239-57

Auerbach D. 1991. Stirring properties of vortex rings. Phys. Fluids A 3:1351-55

Bandyopadhyay PR, Beal DN. 2007. Exception to Triantafyllou's Stroubal number rule of flapping. Presented at Am. Phys. Soc. Div. Fluid Dyn. Meet., 60th, Salt Lake City

Bartol IK, Patterson MR, Mann R. 2001. Swimming mechanics and behavior of the shallow-water brief squid. 7. Exp. Biol. 204:3655-82

Baird MHI, Wairegi T, Loo HJ. 1977. Velocity and momentum of vortex rings in relation to formation parameters. Can. F. Chem. Eng. 55:19-26

Benjamin TB. 1976. The alliance of practical and analytical insights into the nonlinear problems of fluid mechanics. In Applications of Methods of Functional Analysis to Problems in Mechanics, ed. P Germain, B Nayroles, pp. 8-28. New York: Springer

Bernal LP, Kwon JT. 1989. Vortex ring dynamics at a free surface. Phys. Fluids A 1:449-51

Buchholz JHJ, Smits AJ. 2006. On the evolution of the wake structure produced by a low-aspect-ratio pitching panel. 7. Fluid Mech. 546:433-43

Dabiri JO. 2005. On the estimation of swimming and flying forces from wake measurements. F. Exp. Biol. 208:3519-32

Dabiri JO. 2006. Note on the induced Lagrangian drift and added mass of a vortex. F. Fluid Mech. 547:105-13

Dabiri JO, Colin SP, Costello JH. 2006. Fast-swimming hydromedusae exploit velar kinematics to form an optimal vortex wake. F. Exp. Biol. 209:2025-33 
Proposed two-objective protocol to manipulate vortex formation for either maximum efficiency or impulse generation.
Conducted a clinical study of human subjects demonstrating a correlation between optimal vortex formation and function of the cardiac left ventricle.

Observed and modeled optimal vortex formation in piston-cylinder vortex ring experiments.

Experimentally measured the correlation between vortex ring pinch-off and the hydrodynamic efficiency of vortex formation.
Dabiri JO, Gharib M. 2004a. Delay of vortex ring pinch-off by an imposed bulk counter-flow. Phys. Fluids 16:L28-30

Dabiri JO, Gharib M. 2004b. Fluid entrainment by isolated vortex rings. F. Fluid Mech. 511:311-31

Dabiri JO, Gharib M. 2005a. Starting flow through nozzles with temporally variable exit diameter. F. Fluid Mech. 538:111-36

Dabiri JO, Gharib M. 2005b. The role of optimal vortex formation in biological fluid transport. Proc. R. Soc. B 272:1557-60

Didden N. 1979. Formation of vortex rings: rolling-up and production of circulation. Z. Angew. Math. Phys. 30:101-16

Domenichini F, Pedrizzetti G, Baccani B. 2005. Three-dimensional filling flow into a model left ventricle. $\mathcal{F}$. Fluid Mech. 539:179-98

Dong H, Mittal R, Najjar FM. 2006. Wake topology and hydrodynamic performance of low-aspect-ratio flapping foils. 7. Fluid Mech. 566:309-43

Fish FE, Lauder GV. 2006. Passive and active flow control by swimming fishes and mammals. Annu. Rev. Fluid Mech. 38:193-224

Fraenkel LE. 1972. Examples of steady vortex rings of small cross-section in an ideal fluid. 7. Fluid Mech. 51:119-35

Gharib M, Rambod E, Kheradvar A, Sahn DJ, Dabiri JO. 2006. Optimal vortex formation as an index of cardiac health. Proc. Natl. Acad. Sci. USA 103:6305-8

Gharib M, Rambod E, Shariff K. 1998. A universal time scale for vortex ring formation. F. Fluid Mech. 360:121-40

Glezer A, Coles D. 1990. An experimental study of a turbulent vortex ring. 7. Fluid Mech. 211:243-83

Green M, Rowley CW, Haller G. 2007. Detection of Lagrangian coherent structures in three-dimensional turbulence. 7. Fluid Mech. 572:111-20

Haller G. 2001. Distinguished material surfaces and coherent structures in three-dimensional flows. Phys. D 149:248-77

Haller G. 2002. Lagrangian coherent structures from approximate velocity data. Phys. Fluids 14:1851-61

Jeon D, Gharib M. 2004. On the relationship between the vortex formation process and cylinder wake vortex patterns. F. Fluid Mech. 519:161-81

Johari H, Dabiri D, Weigand A, Gharib M. 1996. On the relationship between the formation number and passive scalar pinch-off in starting jets. Proc. 8th Int. Symp. Appl. Laser Tech. Fluid Mech., pp. 399-410. New York: Springer

Kaplanski FB, Rudi YA. 2005. A model for the formation of "optimal" vortex rings taking into account viscosity. Phys. Fluids 17:087101

Kelvin L. 1880. Vortex statics. Philos. Mag. 10:97-109

Kheradvar A, Milano M, Gharib M. 2007. Correlation between vortex ring formation and mitral annulus dynamics during ventricular rapid filling. ASAIO f. 53:8-16

Kilner PJ, Yang GZ, Wilkes AJ, Mohiaddin RH, Firmin DN, et al. 2000. Asymmetric redirection of flow through the heart. Nature 404:759-61

Kim WY, Walker PG, Pedersen EM, Poulsen PK, Oyre S, et al. 1995. Left-ventricular blood flow patterns in normal subjects: a quantitative analysis by three-dimensional magnetic resonance velocity mapping. 7 . Am. Coll. Cardiol. 26:224-38

Krueger PS. 2001. The significance of vortex ring formation and nozzle exit overpressure to pulsatile jet propulsion. PhD thesis. Calif. Inst. Technol., Pasadena. 234 pp.

Krueger PS, DabiriJO, Gharib M. 2006. The formation number of vortex rings formed in uniform background coflow. 7. Fluid Mech. 556:147-66

Krueger PS, Gharib M. 2003. The significance of vortex ring formation to the impulse and thrust of a starting jet. Phys. Fluids 15:1271-81

Lamb H. 1932. Hydrodynamics. Cambridge: Cambridge Univ. Press. 774 pp.

Lim TT, Nickels TB. 1995. Vortex rings. In Fluid Vortices, ed. SI Green, pp. 95-153. Norwell: Kluwer

Linden PF, Turner JS. 2001. The formation of 'optimal' vortex rings, and the efficiency of propulsion devices. 7. Fluid Mech. 427:61-72 
Linden PF, Turner JS. 2004. 'Optimal' vortex rings and aquatic propulsion mechanisms. Proc. R. Soc. B 271:647-53

Maxworthy T. 1972. Structure and stability of vortex rings. F. Fluid Mech. 51:15-32

Maxworthy T. 1977. Some experimental studies of vortex rings. F. Fluid Mech. 81:465-95

Milano M, Gharib M. 2005. Uncovering the physics of flat plates with artificial evolution. 7. Fluid Mech. 534:403-9

Mohseni K. 2001. Statistical equilibrium theory for axisymmetric flows: Kelvin's variational principle and an explanation for the vortex ring pinch-off process. Phys. Fluids 13:1924-31

Mohseni K, Gharib M. 1998. A model for universal time scale of vortex ring formation. Phys. Fluids 10:2436-38

Mohseni K, Ran HY, Colonius T. 2001. Numerical experiments on vortex ring formation. F. Fluid Mech. 430:267-82

Norbury J. 1973. Family of steady vortex rings. F. Fluid Mech. 57:417-31

O'Dor RK. 1988. The forces acting on swimming squid. F. Exp. Biol. 137:421-42

Olcay AB, Krueger PS. 2007. Measurement of ambient fluid entrainment during laminar vortex ring formation. Exp. Fluids 44:235-47

Ongoren A, Rockwell D. 1988. Flow structure from an oscillating cylinder. F. Fluid Mech. 191:197-246

Pozrikidis C. 1986. The nonlinear instability of Hill's vortex. F. Fluid Mech. 168:337-67

Ringuette MJ, Milano M, Gharib M. 2007. Role of the tip vortex in the force generation of low-aspect-ratio normal flat plates. 7. Fluid Mech. 581:453-68

Rosenfeld M, Rambod E, Gharib M. 1998. Circulation and formation number of laminar vortex rings. F. Fluid Mech. 376:297-318

Sallet DW, Widmayer RS. 1974. An experimental investigation of laminar and turbulent vortex rings in air. Z. Flugwiss. 22:207-15

Schatzle PR. 1987. An experimental study of fusion of vortex rings. PhD thesis. Calif. Inst. Technol., Pasadena. $212 \mathrm{pp}$

Shadden SC, Dabiri JO, Marsden JE. 2006. Lagrangian analysis of entrained and detrained fluid in vortex rings. Phys. Fluids 18:047105

Shadden SC, Katija K, Rosenfeld M, Marsden JE, Dabiri JO. 2007. Transport and stirring induced by vortex formation. 7. Fluid Mech. 593:315-31

Shadden SC, Lekien F, Marsden JE. 2005. Definition and properties of Lagrangian coherent structures from finite-time Lyapunov exponents in two-dimensional aperiodic flows. Phys. D 212:271-304

Shariff K, Leonard A. 1992. Vortex rings. Annu. Rev. Fluid Mech. 24:235-79

Shusser M, Gharib M. 2000. Energy and velocity of a forming vortex ring. Phys. Fluids 12:618-21

Taylor GK, Nudds RL, Thomas ALR. 2003. Fluid and swimming animals cruise at a Strouhal number tuned for high power efficiency. Nature 425:707-11

Triantafyllou MS, Triantafyllou GS, Yue DKP. 2000. Hydrodynamics of fishlike swimming. Annu. Rev. Fluid Mech. 32:33-53

Weigand A, Gharib M. 1994. On the decay of a turbulent vortex ring. Exp. Fluids 6:3806-8

Weihs D. 1977. Periodic jet propulsion of aquatic creatures. Fortschr. Zool. 24:171-75

Williamson CHK, Roshko A. 1988. Vortex formation in the wake of an oscillating cylinder. F. Fluids Struct. 2:355-81
Suggested the generality of optimal vortex formation for aquatic propulsion.

Used numerical simulations of vortex ring formation to show the possibility of delaying vortex pinch-off by manipulating the vortex kinematics.

Compiled kinematic data indicating a narrow range of Strouhal numbers used in swimming and flying. 
Von Kármán's Work: The Later Years (1952 to 1963) and Legacy

S.S. Penner, F.A. Williams, P.A. Libby, and S. Nemat-Nasser

Optimal Vortex Formation as a Unifying Principle

in Biological Propulsion

fobn O. Dabiri

Uncertainty Quantification and Polynomial Chaos Techniques in Computational Fluid Dynamics Habib N. Najm

Fluid Dynamic Mechanism Responsible for Breaking the Left-Right Symmetry of the Human Body: The Nodal Flow Nobutaka Hirokawa, Yasushi Okada, and Yosuke Tanaka

The Hydrodynamics of Chemical Cues Among Aquatic Organisms

D.R. Webster and M.7. Weissburg

Hemodynamics of Cerebral Aneurysms

Daniel M. Sforza, Christopher M. Putman, and fuan Raul Cebral

The 3D Navier-Stokes Problem

Charles R. Doering ....

Boger Fluids

David F. Fames

Laboratory Modeling of Geophysical Vortices

G.F.F. van Heijst and H.F.H. Clercx

Study of High-Reynolds Number Isotropic Turbulence by Direct

Numerical Simulation

Takashi Ishibara, Toshiyuki Gotoh, and Yukio Kaneda

Detached-Eddy Simulation

Philippe R. Spalart

Morphodynamics of Tidal Inlet Systems

H.E. de Swart and 7.T.F. Zimmerman 
Microelectromechanical Systems-Based Feedback Control of Turbulence for Skin Friction Reduction

Nobubide Kasagi, Yuji Suzuki, and Koji Fukagata ...

Ocean Circulation Kinetic Energy: Reservoirs, Sources, and Sinks

Raffaele Ferrari and Carl Wunsch

Fluid Mechanics in Disks Around Young Stars

Karim Shariff

Turbulence, Magnetism, and Shear in Stellar Interiors

Mark S. Miesch and Furi Toomre..

Fluid and Solute Transport in Bone: Flow-Induced

Mechanotransduction

Susannab P. Fritton and Sheldon Weinbaum

Lagrangian Properties of Particles in Turbulence

Federico Toschi and Eberhard Bodenschatz

Two-Particle Dispersion in Isotropic Turbulent Flows

Fuan P.L.C. Salazar and Lance R. Collins

Rheology of the Cytoskeleton

Mohammad R.K. Mofrad

\section{Indexes}

Cumulative Index of Contributing Authors, Volumes 1-41 .... 455

Cumulative Index of Chapter Titles, Volumes 1-41 463

\section{Errata}

An online log of corrections to Annual Review of Fluid Mechanics articles may be found at http://fluid.annualreviews.org/errata.shtml 